\title{
Employing digital twins within production logistics
}

Jannicke Baalsrud Hauge HPU/IKAP

Royal Institute of Technology \& BIBA

Södertälje, Sweden/ Bremen, Germany jmbh@kth.se

Yi Li

Fraunhofer-Chalmers Centre for Industrial Mathematics

Gothenburg, Sweden

yi.li@fcc.chalmers.se
Masoud Zafarzadeh

$H P U$

Royal Institute of Technology

Södertälje, Sweden

masoudz@kth.se

Wajid Ali Khilji $H P U$

Royal Institute of Technology

Södertälje, Sweden

wakhilji@ug.kth.se

\author{
Yongkuk Jeong \\ $H P U$ \\ Royal Institute of Technology \\ Södertälje, Sweden \\ yongkuk@kth.se \\ Magnus Wiktorsson \\ $H P U$ \\ Royal Institute of Technology \\ Södertälje, Sweden \\ magwik@kth.se
}

\begin{abstract}
Digitalisation and automation of existing processes are key competitive factors for industry. Still, logistic operations often comprise manual effort, because the movement of goods and material places stringent requirements on the interactions between different systems, human-computer/robot-interaction as well as on changes in the operative processes. In general, the introduction and up-take of new enabling technologies, like the IoT, in complex systems evolved over decades, are challenging. The experience has shown that it is hard to assess all restrictions and interactions between new and old components before any new equipment or infrastructure is implemented and put in operation. This paper presents and discusses the usage of digital twins for supporting the decision-making processes in two different areas: Workstation design and logistics operation analysis. The results are based on tests and experiments carried out in a production logistics test-bed that includes physical devices, an IoT-infrastructure and simulation software. The digital twin is realised in a combination of using Unity and the simulation software IPS. The primary results show that there is no one-size fit all in terms of granularity of the underlying simulation model as well as for the reduction of reality in the digital twin, but the results also indicate that a context-aware digital twin supports the decision-making within a given scope.
\end{abstract}

Keywords-Technology assessment, decision-making, digital twins, cyber-physical system, production logistics

\section{INTRODUCTION AND PROBLEM DESCRIPTION}

Logistics is still a key component in an efficient production with the main task to handle and provide material at the right time, quantity, quality and place. Even though, the automation levels range from fully manual to fully automated and robotized material handling, where the average degree of digitalisation and automation is lower within production logistics than within core production processes $[1,2]$ and the logistics is still characterized by:

- Low automation degree

- Much manual work (often hard)

- Often low usage of advanced technology

However, despite these characteristics, it can be argued that production logistics is a key area in the current shift in manufacturing logic towards an increasingly decentralized, circular and self-regulating value creation, enabled by concepts and technologies such as CPS, IoT, IoS, cloud computing and smart factories. Logistics related operations counts for a significant part of the total cost. According to [3] it varies between 10 and $95 \%$, but often with low added-value to the product or service [4]. Thus in order to reduce the logistics costs as well as for improving the service quality, there is a trend towards technology driven innovations, like automated guided vehicles, diverse intelligent and smart cargo solutions [2], ITS [5] as well as increased servitisation of logistics [6]. By providing material and information at the right place, in the right quality and quantity as well as in right time by using automated guided vehicle (AGV) and Real Time Location System (RTLS), the vision of future manufacturing system ensuring real-time tracking of material flows, a seamless information flow, improved transport handling, energy and material efficiency as well as an accurate risk management can be achieved [7]. Furthermore, the relation of logistics costs vs total costs can be improved.

There are examples of successful implementation of the aforementioned components and technical solutions in an operative environment [8]. Therefore, the main challenges are more related to the implementation of different components in an existing operative environment than on the technical development $[9,10]$. A key challenges is related to assessing how different new components will interact with the existing systems - i.e. a typically challenge when dealing with technology introduction in complex system [6]. The usage of test-beds can contribute to overcoming these challenges and thus several test-beds for advanced technologies implemented in production and logistics have been established [11]. These offer test facilities as well as educational and vocational training to local manufacturing industry and stakeholders involved in the handling of the material and aims at improving the competitiveness of the regional industry [11, 12]. However, even if the physical test-bed allows potential users to experience and test different technologies and solutions in a safe, but realistic environment [14], it will not sufficiently contribute to the understanding the interactions of new components in an industrial operative environment, since there are several context related limitations. One main challenge of logistics systems is the interaction, e.g. HRI (Human-Robot Interaction) and RRI (Robot-Robot Interaction). Conventional solutions for dealing with interaction solutions focus on point-to-point integration while approaches such as enterprise service bus (ESB) have shown great potential to create a more flexible system to increase interoperability among the agents. A second challenge is related to the decentralised decision-making process carried out by the autonomous robots (like automated guided vehicles, or smart cargo) $[2,3,14]$. The usage of digital twins can help in overcoming this challenges and support the decision-making 
process (of the technology assessment) [15] by allowing manipulation of the digital twin to investigate different solutions and the implications on an existing (real physical) environment. It is important to build an overall digital environment where users can become familiar with the replicated objects, explore related processes and assess possible solutions. However, the autonomous decision-making process of the components (like an AGV) remains a challenges, since the algorithm used for the simulations of the potential AGV in the digital twin, not necessarily corresponds to the interaction and the decision the autonomous object makes in the physical environment. This approach also includes the above-mentioned challenges regarding AGV fleet management and interoperability of the agents. $[17,20]$.

The focus of this article is to discuss how digital twins can contribute to support the decision-making process of selection of the right components for a specific company both related to the degree of automation and the digitalisation of the operations. The rest of the article first describes the requirements on a digital twin used for decision-making; secondly, how the manipulation of the digital twin can be used for a better understanding of the system and components interactions, before the test-bed we are using is described in more detailed. Finally, we describe how the different components are integrated and interacting and as well as the first results. The last section discusses the main challenges and the next steps.

\section{NEEDS AND REQUIREMENTS ON A DIGITAL TWIN FOR} PLANNING PRODUCTION LOGISTICS ACTIVITIES

Technology introduction has a large impact on the organisation of operational processes both at managerial and operative level [20]. Therefore, it is not sufficient to only pay attention to the technical aspects, but also required to predict their outcomes and based on that, make right decisions. According to [21] digital twins can support planning as well as prediction of the working processes.

According to [14] there are several explanations of what a digital twin is. For the our purpose, which is to support the decision-making process on technology introduction in existing industrial environment (here realized using a test-bed to reduce the complexity), we build upon the definition by Tao et al. ([14] p.3566) as well as the characteristics of digital twin as described in [14] in respect to a) real-time reflection b) interaction and convergence and c) self-evolution. As described in the introduction, a main challenge of introducing concepts like IoT, CPS, and Industry4.0 etc. in existing environments is the assessment of how the technology will affect the whole organisation and interact with existing solutions. According to $[15,19,20]$ digital twins that can be manipulated can support the decision-making process. Regarding the assessment of autonomous and decentralized decision-making, which is required in cyber-physical systems $[22,24]$ it is hard to model these with the right interactions with other system components and with the right routing (decided upon by the autonomous object) $[2,21]$. However based on experiences on importing sensor data from a labenvironment into the virtual environment, comparing the routes and interaction with the first simulations, and then modify and manipulate these data in the digital twin (by using game mechanics), a better system understanding can be achieved [25].
Digital twin as a concept was established more than a decade ago, but several definitions exist [14]. Digital twin can mirror or simulate complex scenarios very close to the reality $[13,14,24,25]$. Besides providing an opportunity to be used as a learning and awareness-increasing tool in which operators, or management can use virtual reality (VR) tools to experience the real working conditions with high precision [26], the digital twin can support the introduction of new technologies and installations within the existing physical environment [23, 27]. In this case, we simulate the working processes and observe the material movements, machines, robots etc. and their interactions [25]. One of the Swedish test-beds focuses on production logistics, i.e. it focuses on the material and information handling within the production system, with strong and integrated interfaces to supply chain management. This physical test-bed should allow testing of 1) man to material technologies 2) robot to material 3) material to man and 4) material to robot. Furthermore, in order to realise the latter, it should explore the usage of RTLS (Real Time Location System) and the interaction and operation of different AGVs. It should be set up to meet the industrial need for improved demand assessment, dynamic and more efficient milk-runs, shortened cycle times, reduced bullwhip effects, highly transparent and integrated supply chains as well as improvements in production planning, real time information flows, end-to-end supply chain transparency and general improvements in flexibility [7]. By the four pillars of technology support, real-time information, interoperability and decentralized decisions (as shown in Fig 1), core technology areas needed should include real-time data acquisition, organization, and management; digital twin representation; data analytics for logistics applications; and digital twin-based shop-floor management and control [13].

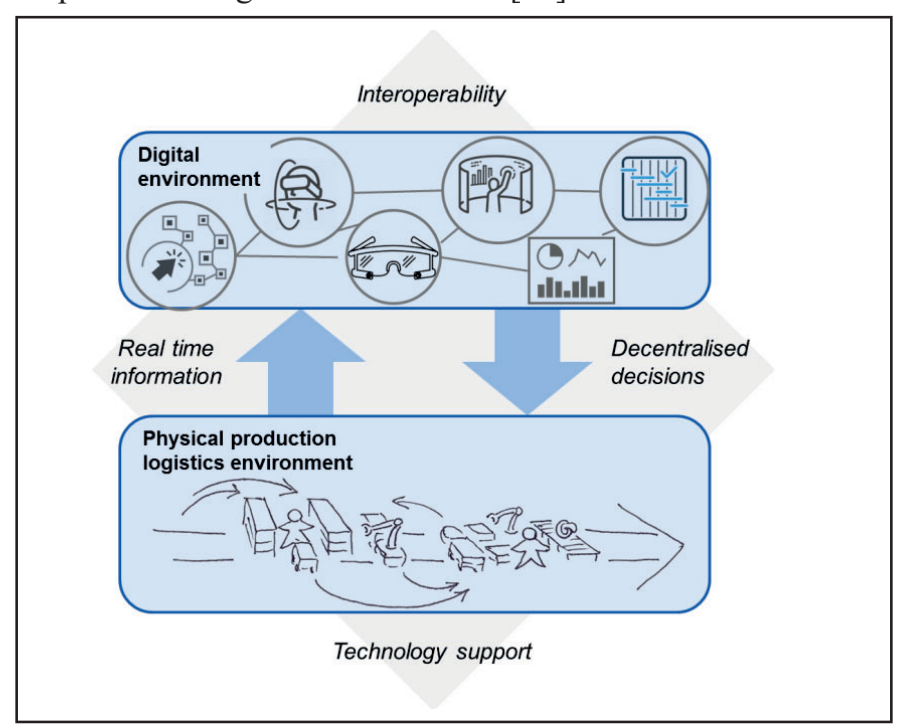

Fig. 1. The digital and physical environment for production logistics, relying on the four pillars of technology support, real-time information, interoperability and decentralized decisions.

\section{The PhysicAl AND Digital TEST-BED}

\section{A. Alignment}

The production logistics test-bed needs to be aligned to the industrial requirements and therefore allow development and testing supporting the supply chain transparency as well as smart factory-internal production capabilities. It needs to include digital and real features linked to both operational 
phases as well as phases of development and engineering. The tests and experiments are related to different configurations of components, connection between using IoT and allow examinations of HMI and HRI interaction. The aim is to highlight how a gradual transition to digitized logistics can take place and allow companies and users to experiment with different combinations in order to find the right level of automation and choose the right technology for their own company, which leads to a more sustainable production.

\section{B. Physical Environment}

A main function is material movement i.e. either that a person or a robot picks up the material or a robot or a person delivers the material [28]. This forms the four fundamental activities of material-to-human, material-to-robot, human-tomaterial and robot-to-material.

The choices of picking and delivery solutions depends on the underlying logistic concept and on other factors like real time monitoring of stock level, tracking of material as well as the quality of the information provided for this decision. How such a solution can be realized in an industrial context depends not only on the physical conditions of the moving material and goods but also on the boundaries given by the building infrastructure [9]. This often leads to a limited possibility in the selection of relevant technologies.

In our first set-up, we have decided to focus on connected equipment and RTLS, since these are a prerequisite for being able to realise a transparent and efficient material flow from one warehouse to a production site. On the long term, we will use the test-bed to develop more explorative solutions for increased automation, leading to a focus on material-to-robot and robot-to-material.
In material-to-robot, material is moved to the robot position manually, automated or semi-automated by using conveyor belts, forklifts, trolleys etc. In this experiment, an AGV delivers the material to the robot station by carrying racks of raw material to the intended position. In robot-tomaterial, the robot moves to the material position and collects the material. Examples can be AS/RS systems (Automated Storage \& Retrieval System), having a robot arm mounted on top of an AGV, or the AGV has the possibility to collect the items. The initial demonstration scenario, as shown in Fig. 3, is to kit three different parts in two different kits. Even though there are many interesting challenges related to the picking process as such (normally it is bin picking), we have left this out and focus currently only on a higher level, which means we need a less granular model for the digital twin. Following items play role in the physical environment (examples shown in Fig. 3):

- Collaborative robot

- $\quad$ Automated guided vehicle (AGV)

- Vision system

- Virtual reality (VR) equipment

- Real time locating system sensors

- Kitting material including parts and boxes

- Transportable racks

- Door

- Elevator

- Connectivity infrastructure including routers

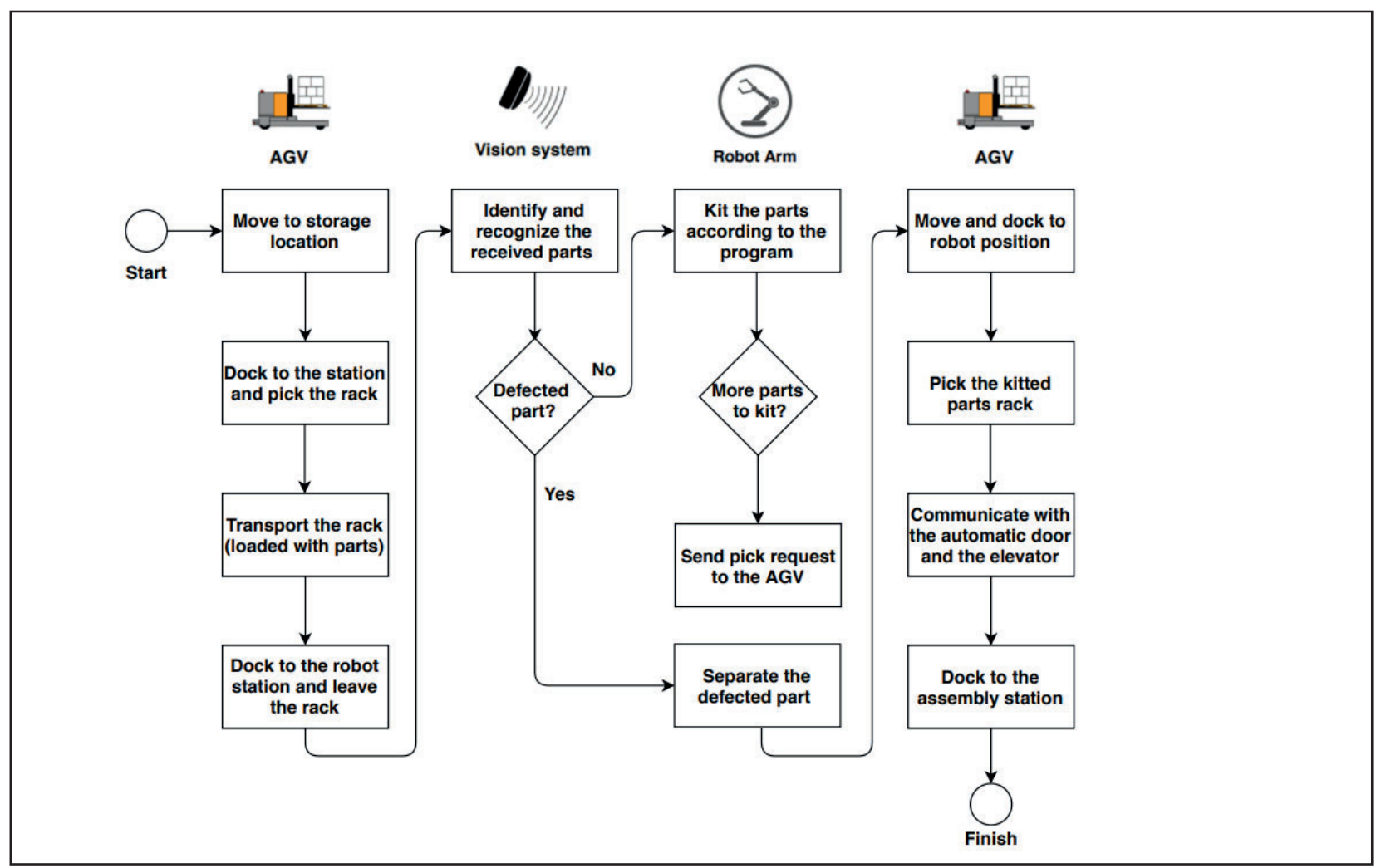

Fig. 2. Envisioned movement for material delivery within the testbed 
As shown in Fig. 2, incoming goods are stored in a storage position, which consists of three transportable racks. The racks are transported to the kitting station by means of an AGV. Raw material/parts will be inspected by the vision system and defected parts will be rejected out of the flow. A collaborative robot does the kitting and the AGV transports the kits to the final destination, which is the manufacturing area, which in our case is an assembly line with nine stations [23]. Since the delivery destination is located on a different floor, the AGV must go through a door and an elevator. Communication between the $\mathrm{AGV}$, door and the elevator needs to be taken into account. By reaching the destination, the AGV leaves the kit rack on predefined position, pick an empty rack and travel back for the next mission. All the racks will be equipped with RTLS sensors to monitor the location of the racks in real-time.

There are some challenges in the realization of this scenario, since it requires interaction with infrastructure parts that are not designed for the purpose, specifically the realization of opening doors, communication with the elevators as well as that we need AGVs with different functionalities.

Ideally, there should be two to three AGVs to realise the scenario as well as to be able to test different types and the interaction between them. Different types of AGVs have different pros and cons, and as a test-bed, it is important that a user of the test-bed can get a good overview of the different functionalities and technical requirements from different $\mathrm{AGV}$ types. Even though the communication protocols between them in many cases are standardized and many AGV providers also offer suitable fleet management software, for instance route optimization algorithms can vary. Therefore, we use the digital twin, both as pure simulation and with data from what we already have in operation to:

- Investigate how we can realise the required interactions between the $\mathrm{AGV}$, the elevator and the door.

- Mission scheduling

- Compare the simulated optimized route in the digital twin to the one the AGV actually chose in the physical environment.

- Investigate how to best realise the part of the physical environment for the quality checkpoints and how to embed this best in the material flow.

\section{Digital environment building the IoT solution}

In order to investigate the problems mentioned in section II, we will integrate the physical objects and the digital twin in a single system, which components communicate via a data streaming bus. This system interconnects all components to make use of digital twin of the real-world scenario. As a first step communication between each component was realised. A data streaming bus, which will hold and serve messages wherever needed was chosen for this purpose. Secondly, there is an application layer, which is required in order to write business logic or calculations. In addition, data storage and digital twin ought to be connected through the application layer. Figure 4 shows the system architecture for the production logistics digital twin.

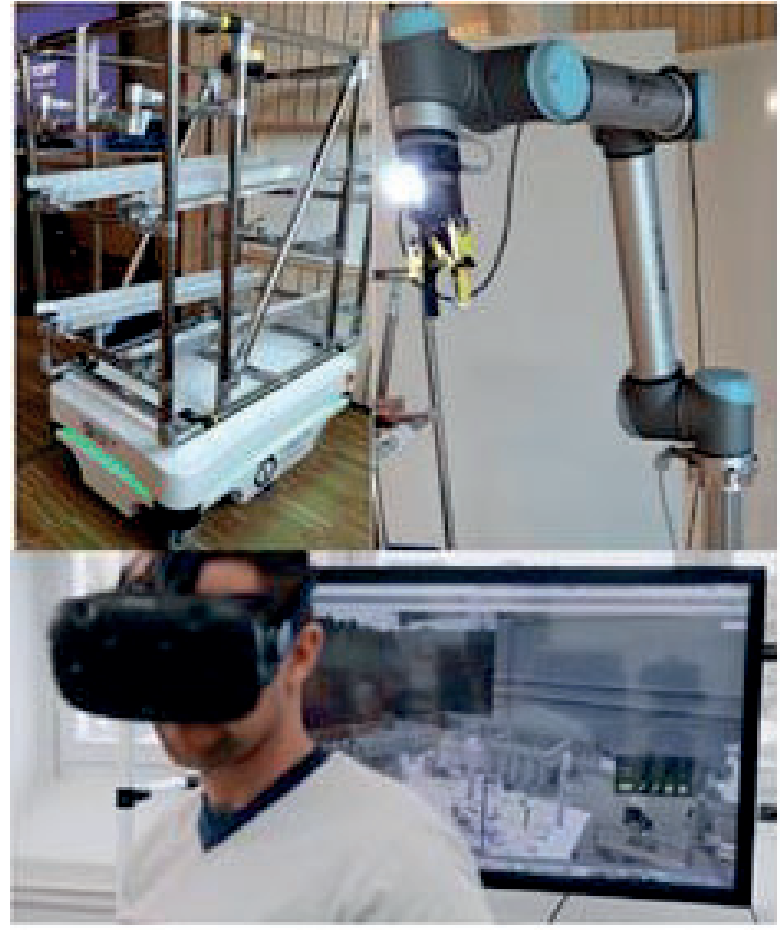

Fig. 3. Examples on physical equipment of the testbed

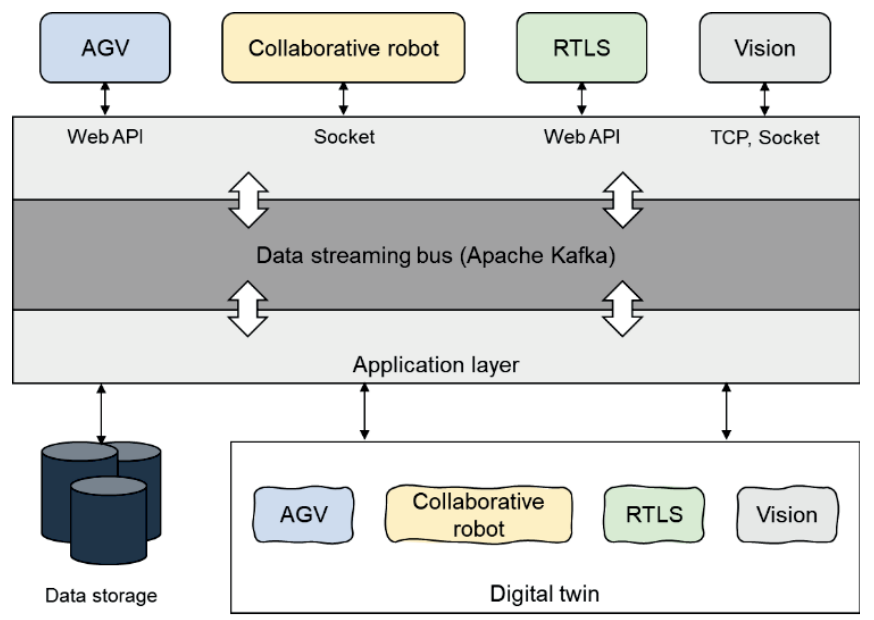

Fig.4: Production logistics digital twin system architecture

In the digital environment, replica components of the physical environment are defined. Each replicated component has to go through the data streaming bus and application layer to send and receive information from the physical environment since the operating systems, data formats, and communication methods are different by each component. The next paragraphs describe communication between the components in more details:

a) The AGV communicates via web API. The real-time location of the AGV can be identified by calling the predefined API from the manufacturer. The results of this API are not only location data, but also various parameters such as battery status, speed, and orientation of the AGV, that may deliver additional valuable information for logistics operations. Of specific relevance is to know that the AGV uses its own coordinate system. This is translated into the digital twin model to align it with the physical environment. Since the coordinate system in the digital 
twin perfectly align with the real world in meters, coordinates in meters and angular rotation in degrees are used.

b) The collaborative robot uses a pre-defined language to program and communicate with the external world. The robot can be programmed in several ways. In this case, it is used to communicate over a TCP socket connection, in which the robot is treated as a server and connected via a TCP client application. The robot sends six angular rotations to the unity model (digital twin), for simulation.

c) The RTLS is used to identify various location of the components in an indoor location system. This system is used to track objects like parcel/packages, tray's, tables or any movable object for that matter. The RTLS system uses API to retrieve location data of these various objects.

d) The vision system is currently built by using Microsoft Xbox Kinect. It will at a later stage be replaced by a 3D camera. The vision system is currently working via a $\mathrm{C \#}$ program with the help of OpenCV wrapper, called EMGU. So far, we are using Haar classifier method for deep learning mechanism to identify an object. Haar algorithm requires thousands of positive and negative images to train a classifier, which is time consuming. In later stages, we plan to work on Point clouds, a solution that is more robust. The vision system elaborates coordinates from a recognized image, which Xbox Kinect contains in properties of an image object that have $\mathrm{X}, \mathrm{Y}$ and $\mathrm{Z}$ coordinates. Those are then sent to middle-ware that translates into the collaborative robot coordinates.

e) The digital twin is built using standard assets in Unity, along with some complex prefabs like AGV and collaborative robot, delivered by the supplier or modelled using IPS Each controller is responsible for fetching data or controlling the machine. The Digital twin can visualise the track of AGV to analyze deviations.

f) Data storage is used for the long-term data solution. This will help build a possibility to replay a particular scenario. For example, simulating 1-hour run time of AGV and collaborative robot in digital twin. Message broker can be used instead, but later there will be a need to store data for longer period of time, for data analytics, or data mining. This layer is important for many reasons. One of the reasons is to unify the data transport layer. More importantly, it provides possibility of replication. In case of natural disaster or loss of hardware, a replication will be used or a failover cluster can be configured at different location. In addition, there will be single point of data disposal and retrieval. Which makes it easier for many other devices, who need data from a single point.

We have set up a scenario to apply and test the digital twin model to general situations in production logistics. in this scenario, the Cobot picks up the box containing the articles and delivers it to the $\mathrm{AGV}$, and the AGV transports the box to the quality check station. Fig. 5 shows the workflow of this scenario.

The next section describes the different possibilities we have for the models in the digital twin, as well as outlines what we currently mainly look at.
[AGV] Wait at the charging station

[AGV] Move to the waiting station

[AGV] Wait at the waiting station

$\downarrow$

[Cobot] Start box picking mission

[Cobot] Pick the box from table to AGV

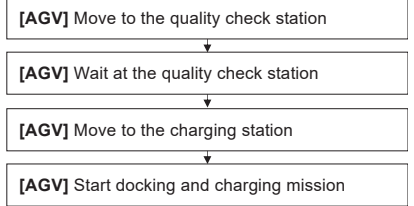

Fig.5. Workflow diagram of the production logistics scenario using digital twin model

\section{UsE OF THE Digital TwIN}

The digital environment does not only mirror the physical environment in the test-bed, but also allow 'manipulation' to allow experimentation with different technologies and equipment prior to the decision in a virtual world.

\section{A. Applications for workstation design}

As described above, the digital twin mirror a physical twin, but as typically for simulation environments, it does not necessarily need to mirror every single process at all level, but only those being required [22] - in our case processes for making decisions. Hence, how detailed and our models are, depends on what we intend to use them for. For the purpose of the test-bed and the given requirements, we need different granularities for different manipulations, i.e. if we look at the interaction of the $\mathrm{AGV}$ and the picking robot in the picking process, we need more detailed models than if we look at the material flow from the 'warehouse' to the assembly area. Therefore, we will give three examples in this spectrum of applications, in this case focusing the (1) engineering of workstations, (2) layout planning and (3) collaborative robotics. This will cover all the levels we need in the test-bed on long term and gives an overview of the different levels of granularities we need.

(1) In the case of workstation design, a Digital Human Modelling (DHM) tool IMMA (Intelligently Moving Manikins) is used [28]. This is used to analyse human postures and motions in order to evaluate both human-product interactions and human-production system interactions and then predict ergonomics issues before the product and workplace exist physically. In IMMA, not only a biomedical model, but also a collision model using hierarchies of rectangular swept spheres (RSS) [12,30] represents a manikin. A reaching movement can be achieved by simply specifying a sequence of target positions that the fingertip has to trace. In Fig. 6, the manikin's left hand reaches deep into the engine bay of a truck to replace a headlight bulb by following a computergenerated path for a sphere [30] where the tip of a hand's middle finger was used as the manikin's end-effecter.
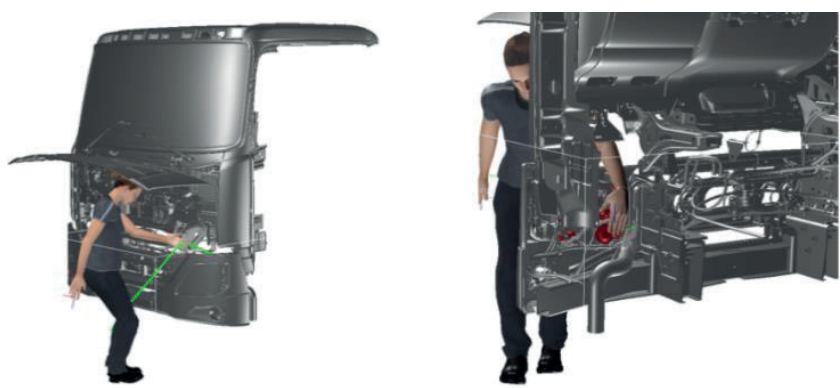

Fig.6. Truck headlight bulb replacement simulation. The path in green connects the initial position and the goal position. Before approaching the truck, the manikin was in a standing pose with feet together and arms by the sides. Truck CAD model courtesy of AB Volvo. 
We use a novel hierarchical controller system for reducing the time-consuming set-up [31]. The result is a sequence of ergonomically sound and collision-free motions that accomplish a task. The framework is demonstrated on one industrial test case that requires the utilization of support. As the manikin walks to the car in Fig. 7, the manikin puts the left hand on the chassis to maintain the balance before inserting a box to its final position.
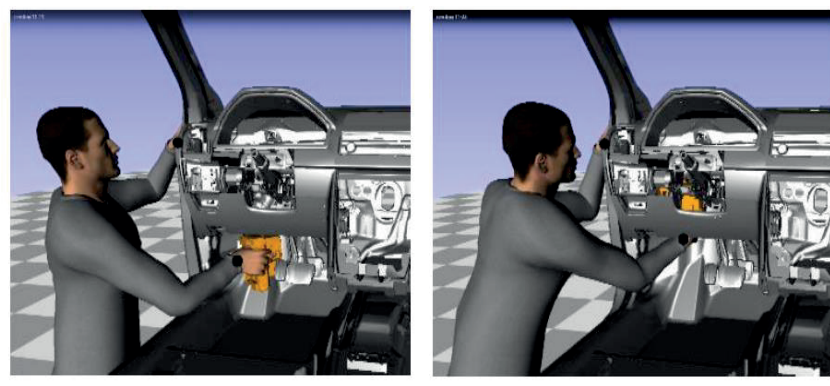

Fig. 7. Simulation of assembling the Central Electronic Module (CEM). Car CAD model courtesy of Volvo Cars.

(2) To support a virtual layout process, we use a novel digital factory layout tool that optimizes both the layout of machines and the corresponding ergonomic logistics considering space constraints [29]. The ergonomic logistics is calculated using the IMMA (Intelligently Moving Manikins) manikin [28].

(3) For the case of human-robot collaboration, the fidelity of the simulation is affected by how accurate the motions of the robot can be described. However, accurate simulations of industrial robots are only available through the manufacturers' proprietary offline programming software or through the limiting standardized Realistic Robot Simulation (RRS) interface [32]. By developing a virtual representation of the robot and an interface to the real robot, a robot's simulation capabilities were unlocked [33]. The ability to synchronize the simulation to the physical robot (Fig 8) through IO signals enables the execution of possible optimization algorithms based on the virtual representation with the initial state from the real robot. When the robot moves in the physical world, the simulation software can update the virtual representation of robot. On the other hand, after a successful simulation, the robot program can be sent to the real robot and then executed by the robot. The resulting trajectory from the real robot is sent back to the simulation software and can be replayed if desired. One example is shown in Fig. 9, where the robot goes from a state outside the cage to a state inside the cage. The motion between the two states is simulated beforehand and verified before it is executed on the real robot.
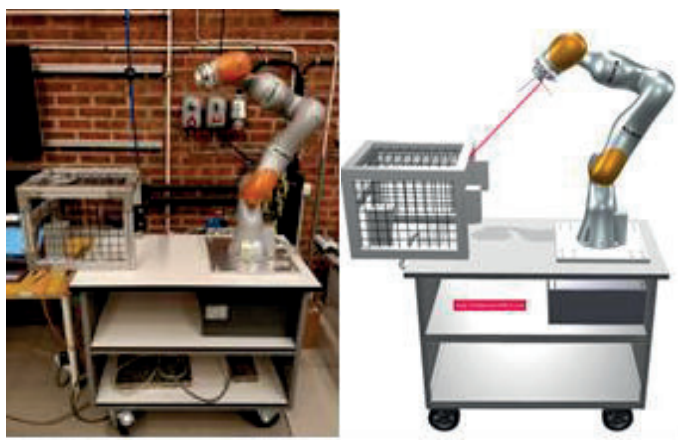

Fig. 8. Physical robot (left) and its virtual representation (right)
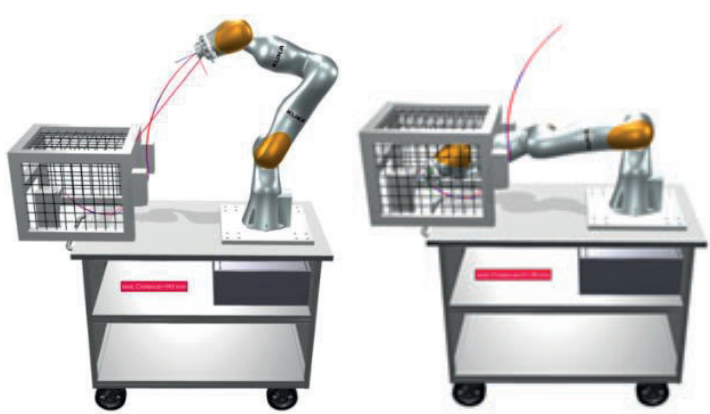

Fig. 9. The robot's start state (left) and end state (right).

\section{B. Applications for logistics operations analysis}

As described in the introduction, one main challenge with technology introduction of new technologies in existing, operating environments is to assess both the interaction and interference with existing systems as well as to decide upon the right new object (like the type of AGV). Secondly, as described above a challenge is that there is limited information about the algorithm the AGVs are using for calculating their path in the physical environment. In our case, we have however full control over the algorithm the digital twin of the AGV (modelled using IPS), which is the optimised algorithm. Comparing the results of the digital twin optimised algorithm and the physical movements of the AGV can give valuable information on the behaviour and thus help in the assessment of the suitability for implementation in different environments. Furthermore, the integration of the physical data in the digital twin allows a better visualisation that can be used for different purposes:

a) Detailed investigation of the exact movements of the AGV or collaborative robot (as seen in fig. 10). Using a re-play function as well as combining different experiments in one single digital twin scenario helps to understand the variation in movements as well as to recognise patterns (i.e. continuously occurring deviations between real movements and optimised calculated paths).

b) To explore an environment to which you might have no access (either using VR, AR or a 3D computer model on the PC). This can be used for teaching purposes to explain the interaction of the different components as well as for letting the students try out simple actions in a safe environment. Fig. 10 illustrates this possibility. The first picture shows the digital model of the lab environment. The viewer can use a camera to move around and experience the room. The second image shows the detail of the models- here the path of the AGV is visualised. The last image shows the movement of the physical AGV (data imported in the Unity model via the communication bus). 

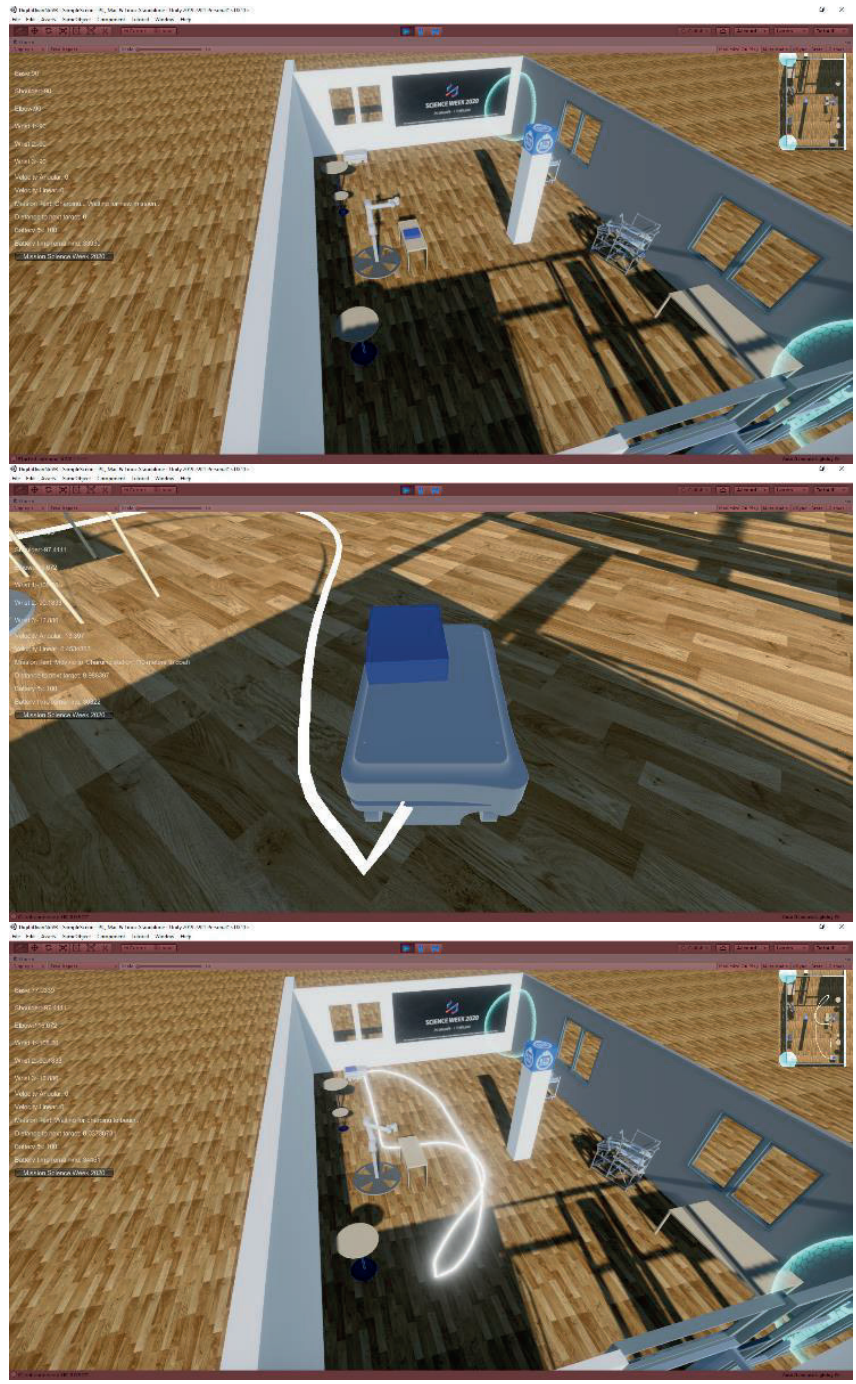

Fig. 10. Tracking AGV in real time within the digital twin

The digital twin has the capability to start the different physical operations via the digital twin interface. This open up the possibility for carrying out experiments in risk areas (or in our case) giving access to those personnel that do not have the right safety level to operate the physical equipment on site, or for individuals who cannot attend the physical lab for any reason. This increases the possibilities to utilise expensive lab equipment to a higher degree. Even though this will reduce the safety risks for personnel operating in the physical environment, it might cause other risks such as moving objects around the lab with no real visual control over the lab environment.

The mixed-reality environment which we have described here is in its prototypical implementation phase and the results are preliminary, but promising. During the last semester, the lab used as a part of a master course on production logistics. Here a group of students carried out experiments to study movements of the AGV in the physical lab. At that time, it was not possible to import and store the data in the digital twin for visualisation, but the collected data will now be imported and compared with the ideal (optimised) movements. Secondly, the first tests of using the digital twin for educational purposes have been successfully tried out in different labs. We have therefore now integrated our digital physical twin environment for educational purposes in a digitalisation course that will start in the spring term. The remote control scenario is so far working well as long it is operated within the network (security) issues. In a next step it will also be possible to operate it from outside. This will allow us to connect different test-beds to one single digital test-bed in order to look at larger challenges.

\section{DISCUSSION AND CONCLUSION}

Digitalisation and automation of existing processes are key competitive factors for industry. Still, logistics operations often comprise manual effort, mainly due to the complexity of the interactions between different systems, humancomputer/robot-interaction as well as on changes in the operative processes. The main objective of this paper was to investigate and discuss how digital twins can contribute to support the decision-making process of selection of the right components for a specific company both related to the degree of automation and the digitalisation of the operations. Our first experiments indicates that within the application area of workstation design, the granularity of the modelling is of utmost importance. The examples discussed in this article-i.e. the interaction of the UR robot and the $\mathrm{AGV}$ in a picking task, and the usage of $\mathrm{AGVs}$ for moving material from $\mathrm{A}$ to $\mathrm{B}$, illustrates this very well. In the first case, the robot-robot interaction, a main decision is related to the flexibility of the two components in gripping specific components, the time it takes to pick (i.e. identify the component, grip it, put it in the right place), and the accuracy both of the picking process itself, as well as on the docking process of the AGV. The main "competitor" would in many cases be a human that is currently much faster and more flexible, but perhaps less accurate, and cannot pick $24 \mathrm{~h}$. It would therefore be necessary to mirror the physical process so detailed that a comparison is allowed. In addition, the digital twin might be used for investigating topics related to human-robot interaction and other issues related to the picking process. Using a digital twin for decision-support for this purpose requires that the information that $\mathrm{ab}$ is very similar to the physical (real) process, but from other perspectives. In this case the cost and time factors are less relevant, whereas the exact mapping of the movements of the object interacting with the human would be of relevance. As described above, this do require specific software solutions as well as deep knowledge in how to map this correctly. In both cases, a very granular modelling and a simulation that is exact and correct is prerequisite in order to use the DT as decision-support tool. The second case shows more how a DT can be used for investigating logistical operation, both at a planning level as well as in order to better understand the decision-making and underlying algorithm of automated vehicles, like the AGV and the UR robot. In the area of analyzing logistical operations, we explained two different cases. In the first of these, we are interested in improving the understanding of an existing object (AGV) which autonomously decide how to get from A to B and how it reacts to obstacles. For this, accuracy of the mirrored environment in which we can import the location data (see fig.9) is needs to be medium high (not as granular as in the workplace design) and then to use a replay function for comparing the physical path with the optimized path. Based on this comparison, we may decide on how the layout should be, and also use it for deciding what type of AGV that seems more suitable to be operated in environments with more or 
less interaction with other moving objects/subjects. The limitation for this, is that we can import the data in a model, and that this model (if it is like in our case a testbed), delivers a sufficient similar environment as the environment for which we want to implement the AGV. The second case was to use the digital twin to explore a restricted area. Here, depending on what we will investigate it is often sufficient to have a low accuracy in the mapping.

These first results of how a digital twin can be used to support the decision-making process within production logistics processes are quite promising, but in order to be more explicit in what is meant with high, medium and low accuracy in the different context, more tests and experiments with different objects are necessary. Furthermore, we still have to explore the boundaries and opportunities of using a digital twin for at a higher strategic and planning level.

\section{ACKNOWLEDGMENT}

The present work was carried out partly within the project DIGILOG (Produktion 2030, Vinnova) and UniLog (Central Baltic program, grant no. CB743). This presentation does not represent the opinion of the European Community or Vinnova, and the European Community and Vinnova are not responsible for any use that might be made of its content.

\section{REFERENCES}

[1] BVL, "Digitisation in Logistics",2016. [Online]. Accessible: file://C:/Users/jmbh/Downloads/BVL17_Position_Paper_Digitisation in Logistics.pdf.

[2] M.Forcolin et al, "EURIDICE- IoT applied to Logistcst using the intelligent Cargo concept," in Thoben, K-D; Stich, V. \& Imtiaz, A. ICE proceedings, 2011.

[3] P.Veres et al, "Intelligent Transportation Systems to Support Production Logistics", in Jármai K., Bolló B. (eds) Vehicle and Automotive Engineering. Lecture Notes in Mechanical Engineering. Springer, Cham, 2016.

[4] M. Christopher, "Logistics and Supply Chain management," $5^{\text {th }}$ edi, 2016.

[5] T.Zunder et al, "Is it Possible to Manage and Plan Co-Modal Freight Transport Without a Centralised System?," International Journal of Applied Logistics (IJAL) 3(2), 2012.

[6] J.O. Strandhagen et al, "Logistics 4.0 and emerging sustainable business models," Adv. Manuf. 5: 359. 2017.[Online]. Available: https://doi.org/10.1007/s40436-017-0198-1.

[7] E. Hofmann, m. Rüsch, "Industry 4.0 and the current status as well as future prospects on Logistics," Computers in Industry 89, 2017, pp.2334.

[8] S. Jeschke et al, "Industrial Internet of Things and Cyber Manufacturing Systems," in Jeschke S., Brecher C., Song H., Rawat D. (eds) Industrial Internet of Things. Springer Series in Wireless Technology. Springer, Cham, 2017.

[9] Y.Patil, "6 key IoT Implementation Challenges for Enterprises to consider,"2018.[Online].Available:https://www.saviantconsulting.com /blog/iot-implementation-challenges-enterprises.aspx.

[10] I. Ek, M. Wiggberg, and P. Frykblom, Hur kan staten främja användandet av digitaliseringens möjligheter $i$ näringslivet? 2, Östersund, 2018.

[11] H. Bosson, J. Ingmarsson, Digitalisering av svensk industrikartläggning av svenska styrkor och utmaninger, Roland Berger $\mathrm{AB}$ ,2016.

[12] 2018.[Online]. Available: https://produktion2030.se/unikt-samarbetemellan-industri-och-akademi-inom-testbadd-smart-produktion/.
[13] C. Zhuang et al, "Digital twin-based smart production management and control framework for the complex product assembly shop-floor". The International Journal of Advanced Manufacturing Technology, 96(1-4), 1149-1163.,2018.

[14] F. Tai et al, "Digital twin driven product design, manufacturing and service with big data," Int. J. of Adv. Manuf. Techn. 94:3563-3576X, 2018.

[15] B. Brenner, V. Hummel, "Digital twin as enabler for an innovative digital shopfloor management system" in the ESB logistics Learning Factory at Reutlingen University, Procedia Manufacturing 9,2017, pp.198-205.

[16] Aberle et al, "Fabrik Industrie 4.0 - Potentiale, Nutzen und GoodPractice-Beispiele für die hessische Industrie," Meisenbach Verlag Bamberg, 2015.

[17] Uhlman et al, "The digital twin: Demonstrating the potential of real time data acquisition in production systems," Procedia Manufacturing 9 ( 2017 ) 113 - 120, 2017.

[18] H. Warmlink et al, "Gamification of production and logistics operations,"JBR,2018[Online]. Available:https://doi.org/10.1016/j.jbusres.2018.09.011.

[19] A.Ştefan et al, "Approaches to Reengineering Digital Games," ASME. International Design Engineering Technical Conferences and Computers and Information in Engineering Conference, vol 1B: 36th Computers and Information in Engineering Conference, 2016. [Online].Available:V01BT02A051.doi:10.1115/DETC2016-60061.

[20] J. Baalsrud Hauge, "An educational framework for supporting the implementation of the Intelligent Cargo concept",International Journal of Advanced Logistics,5:2,pp.86-100,2016.[Online].Available:DOI: 10.1080/2287108X.2016.1185317.

[21] F. Yao, A. Keller, M. Ahmad, B. Ahmad, R. Harrison and A. W. Colombo, "Optimizing the Scheduling of Autonomous Guided Vehicle in a Manufacturing Process," 2018 IEEE 16th International Conference on Industrial Informatics (INDIN), Porto, pp. 264-269, 2018.

[22] VDI-Richtlinie, VDI 3633 Simulation von Logistik-, Materialfluss- und Produktionssystemen - Begriffe.

[23] J. Baalsrud Hauge et al, "Bridging educational and working environments through pervasive approches", in M Alcaniz et al (2017) LNCS 10622, pp.296-307, 2017.

[24] P. Nokelainen, T. Nevalainen, K. Niemi, "Mind or Machine? Opportunities and Limits of Automation," The Impact of Digitalization in the Workplace. Springer, Cham, 21 pp.13-24, 2018.

[25] J. David, A. Lobov and M. Lanz, "Leveraging Digital Twins for Assisted Learning of Flexible Manufacturing Systems," 2018 IEEE 16th International Conference on Industrial Informatics (INDIN), Porto, pp. 529-535, 2018.

[26] V. Toivonen et al, "The FMS Training Center - a versatile learning environment for engineering education", Procedia Manufacturing, Vol. 23, 2018,pp. 135-140.

[27] HC Pfohl, "Produktionslogistik", in Logistiksysteme, Springer Vieweg, Berlin: Heidelberg, 2018

[28] D. Högberg et al, "Creating and shaping the DHM tool IMMA for ergonomic product and production design," in International Journal of the Digital Human, vol. 1, No. 2, 2016.

[29] E. Larsen, "Fast distance queries with rectangular swept sphere volume," in Proceedings of the 2000 IEEE International Conference on Robotics and Automation, 2000 .

[30] Y. Li et al, "On motion planning for narrow-clearance assemblies using virtual manikins," in Proceedings of the 51st CIRP Conference on Manufacturing Systems, Stockholm, 2018.

[31] W. Kühn, "Digital factory - integration of simulation enhancing the product and production process towards operative control and optimization," in International Journal of Simulation, Systems, Science and Technology, 2006.

[32] R. Bernhardt, "Realistic robot simulation," in Computing and Control Engineering Journal, Vol. 6, No. 4 (1995).

[33] Larsen, C, "Including a Collaborative Robot in Digital Twin Manufacturing Systems," master's thesis, 2018.

X.Stock, Selig, "Opportunities of Sustainable Manufacturing in Industry 4.0,” Procedia CIRP 40,pp. 536 - 541, 2016. 